\title{
Cepheid Limb Darkening Models for the VLTI
}

\author{
Massimo Marengo, Margarita Karovska, Dimitar D. Sasselov and \\ Costas Papaliolios \\ Harvard-Smithsonian Center for Astrophysics, Cambridge, MA, USA \\ J.T. Armstrong \\ Remote Sensing Division, Naval Research Laboratory, Washington, DC, USA \\ Tyler E. Nordgren \\ Dept. of Physics. University of Redlands, Redlands, CA, USA
}

\begin{abstract}
We present a new method to compute wavelength- and phase-dependent limb darkening corrections for classical Cepheids. These corrections are derived from hydrodynamic simulations and radiative transfer modeling with a full set of atomic and molecular opacities. Comparison with hydrostatic models having the same stellar parameters show a larger limb darkening for most phases in our models, and temporal variations related to the hydrodynamics of the stellar pulsation. We assess the implications of our results with respect to the geometric Baade-Wesselink method, which uses interferometric measurements of Cepheid angular diameters to determine their distances and radii. The relevance of the hydrodynamic effects predicted by our model on the limb darkening of pulsating Cepheids is finally discussed in terms of the predicted capabilities of the VLTI.
\end{abstract}

\section{Introduction}

The period-luminosity (PL) relation of Classical Cepheids, since its discovery by Leavitt (1906), has played an important role in setting the extragalactic distance scale. The reliability of this relation, however, depends on the accurate calibration of its zero-point. This is difficult to accomplish, due to the low density distribution of Cepheids in our Galaxy. The determination of the PL relation zero point from geometric parallaxes has been attempted using the database of the Hipparcos mission (Feast \& Catchpole, 1997), but large uncertainties do not allow an unambiguous interpretation of the results (Madore \& Freedman, 1998). The same is also true for the classical Baade-Wesselink (BW) method (Baade, 1926; Wesselink, 1946), which suffers from large uncertainties related to the source colors and metallicity.

The recent advances in interferometry, however, have finally allowed the direct detection of pulsations in nearby Cepheids (Lane et al., 2000; Kervella et al., 2001; Lane et al., 2002). This result have opened the way for a geometric variant of the BW method (Sasselov \& Karovska, 1994), allowing an independent distance measurement of pulsating stars, based on the interferometric determination of their radial pulsations.

(c) 2018 Kluwer Academic Publishers. Printed in the Netherlands. 
As pointed-out by Sasselov \& Karovska (1994), the feasibility of the geometric BW method requires accurate predictions for the limb darkening (LD) of the pulsating star, necessary to convert the measured interferometric visibilities into angular diameters. In this paper we present our time- and wavelength-dependent LD models, based on hydrodynamic models of the classical Cepheids, and we discuss their importance for future VLTI observations of this type of pulsating stars.

\section{Dynamic models for Pulsating Cepheids}

In Marengo et al. (2002) we presented a new method for computing accurate time- and wavelength-dependent center-to-limb brightness distributions for classical Cepheids. This method is based on hydrodynamic simulations of the Cepheid atmosphere, performed in non-LTE conditions with a simplified radiative transfer (Sasselov \& Lester, 1994). The pulsations are induced by perturbing the model atmosphere with a piston, parametrized by matching the radial velocity curve, phase lags and other stellar observables. On top of the hydrodynamic atmosphere, we solve the full LTE radiative transfer problem, using the complete set of atomic and molecular opacities provided by the ATLAS/SYNTHE program (Kurucz, 1970, 1979, 1993). From the SYNTHE emergent spectral intensity, we derive the LD of the pulsating star at a number of wavelengths and pulsational phases.

The center-to-limb intensities from which the LD is derived are computed with SYNTHE for a model atmosphere having (1) the effective temperature $T_{\text {eff }}(\phi)$ obtained as a function of the pulsational phase from observations (Krockenberger et al., 1997), (2) $\log g, L / H$, metallicity, $v_{t u r b}$ and the atmospheric Rosseland opacity from a hydrostatic model approximating the hydrodynamic atmosphere at each pulsational phase, and (3) $T(r), n_{e}(r)$ and $P(r)$ from the hydrodynamic model, excluding the chromospheric region. The main limitation of this procedure resides in the opacities being computed with a hydrostatic atmosphere in LTE conditions. However, the resulting limb darkened intensities are still much improved with respect to competing models, usually derived for static yellow supergiants, since the thermodynamic state of the atmosphere is completely set by the hydrodynamics.

The radial profile of the hydrodynamic atmosphere can be very different from the structure of a hydrostatic atmosphere with equivalent $T_{\text {eff }}$ and $\log g$. This is especially true for pulsational phases characterized by the propagation of hydrodynamic instabilities or shocks, opposed to other phases where the structure of the pulsating atmosphere is quasi-static. The fact that the atmosphere structure changes 


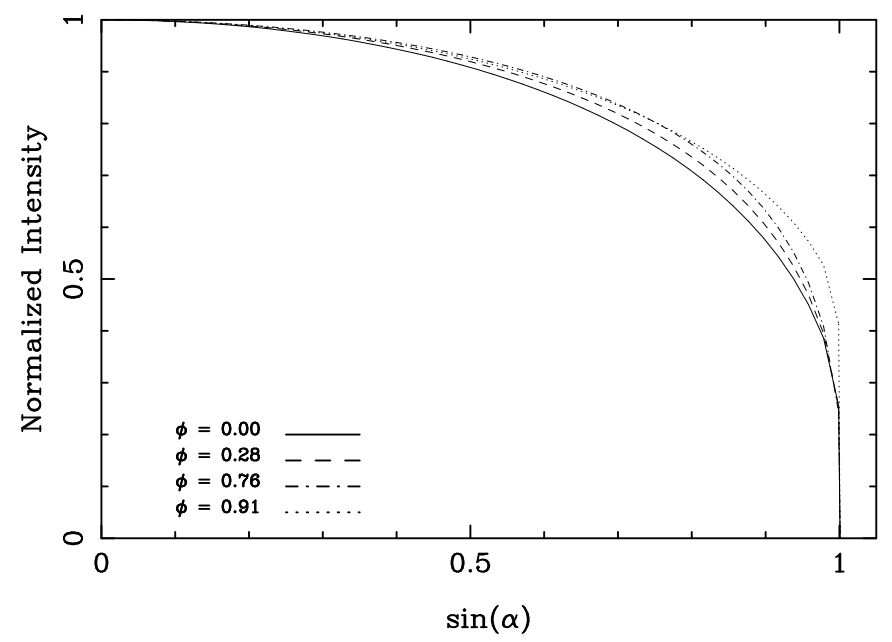

Figure 1. Limb-to-center profiles for $\zeta$ Gem at dynamical phases 0 (minimum radius), 0.28 (maximum luminosity), 0.76 (minimum luminosity) and 0.91 (passage of a shockwave through the photosphere). The profiles are computed for $\lambda=500 \mathrm{~nm}$. The $\mathrm{x}$-axis coordinate $\sin (\alpha)$ is the projected radial coordinate of the star.

as the Cepheid pulsates, leads to different amount of limb darkening for different pulsational phases. Figure 1 shows how the photosphere of the classical Cepheid $\zeta$ Gem appears to be more limb darkened near the minimum radius, and less limb darkened as a consequence of a shockwave perturbing its atmosphere.

The center-to-limb intensities shown in Figure 1 can be used to derive the LD correction $k(\lambda, \phi)$ for the modeled Cepheid. This correction is defined as the ratio between the angular diameter measured by fitting the interferometer visibilities with a uniform brightness disk (UD) model, with the true diameter of the limb darkened star (the "limb darkened diameter" ): $k(\lambda, \phi)=\theta_{U D}(\lambda, \phi) / \theta_{L D}(\phi)$. The LD correction is wavelength and phase-dependent, as a consequence of the wavelength dependence of the atmospheric opacities, and because of the changes in the atmospheric structure as the star pulsates.

As an example, Figure 2 shows the spectral dependence of the LD correction for our model of $\zeta$ Gem at minimum radius. The limb darkening is larger at UV and optical wavelengths than in the infrared. Neglecting to correct for LD in the near-IR, however, will still produce an error of the order of $\sim 2 \%$ with respect to the UD diameter of the star. Note that the LD correction $k$ is very sensitive to the photospheric spectral lines, which can thus be used as an effective diagnostic tool to better constrain the LD with interferometric observations.

Figure 3 shows the variations of the LD corrections with the pulsational phase, in the visible and at near-IR wavelengths (H-band). 


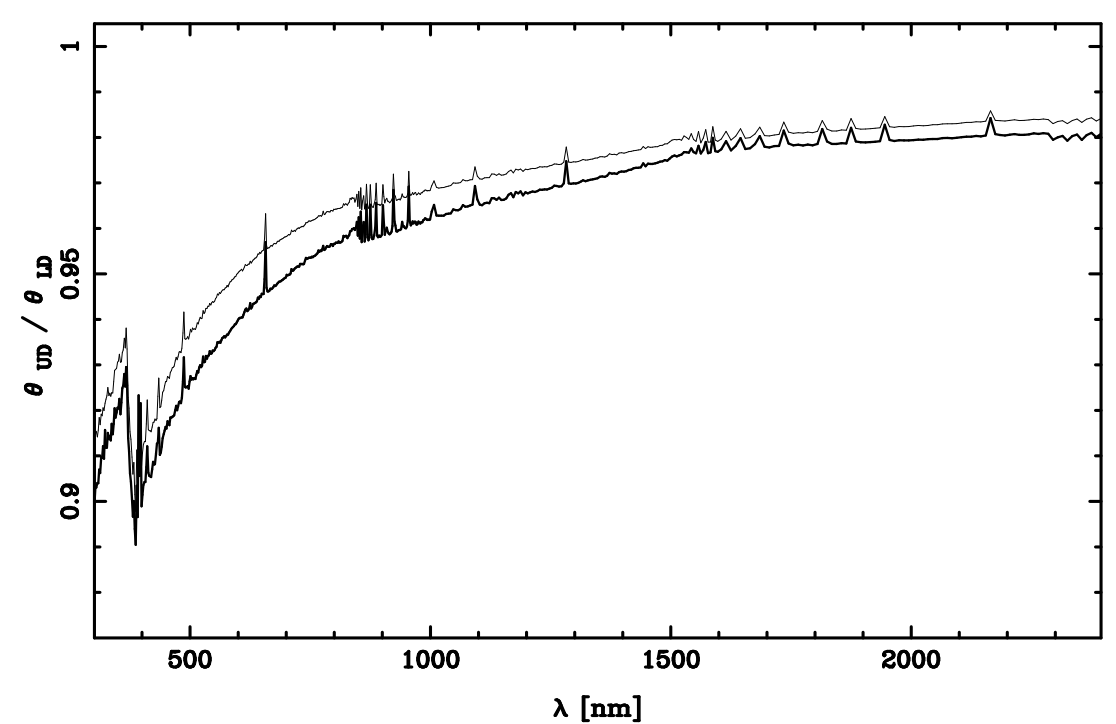

Figure 2. Wavelength dependence of the LD correction $k(\lambda)$ for $\zeta$ Gem at minimum radius. The solid thick line is $k(\lambda)$ from our hydrodynamic model; the thin line is derived from a hydrostatic model having $T_{\text {eff }}$ and $\log g$ from observations.

The dotted lines are the LD corrections for of equivalent hydrostatic atmosphere, having the same $T_{\text {eff }}$ and $\log g$ than the hydrodynamic models. The hydrostatic LD corrections closely follows the changes of $T_{\text {eff }}(\phi)$ with the pulsational phase. In the hydrodynamic atmosphere, however, $T_{\text {eff }}$ is not the dominant parameter. The hydrodynamic LD correction is instead characterized by sudden variations due to shocks traveling through the photosphere. Even when shocks are absent, the LD correction is still strikingly different from the one predicted by an hydrostatic model, as it is fully determined by the time-dependent structure of the expanding/contracting atmosphere. This dependence from the hydrodynamics results in a larger LD of the hydrodynamic models with respect to hydrostatic ones.

\section{Limb darkening Corrections for the VLTI}

The VLTI interferometer, equipped with the near-IR camera AMBER will be especially suited to measure accurate distances of southern Cepheids with the geometric Baade-Wesselink method. To obtain the required accuracy, however, the interferometric measurements should be accompanied by a detailed modeling of the observed stars, taking into account the hydrodynamic effects induced by the pulsations. 


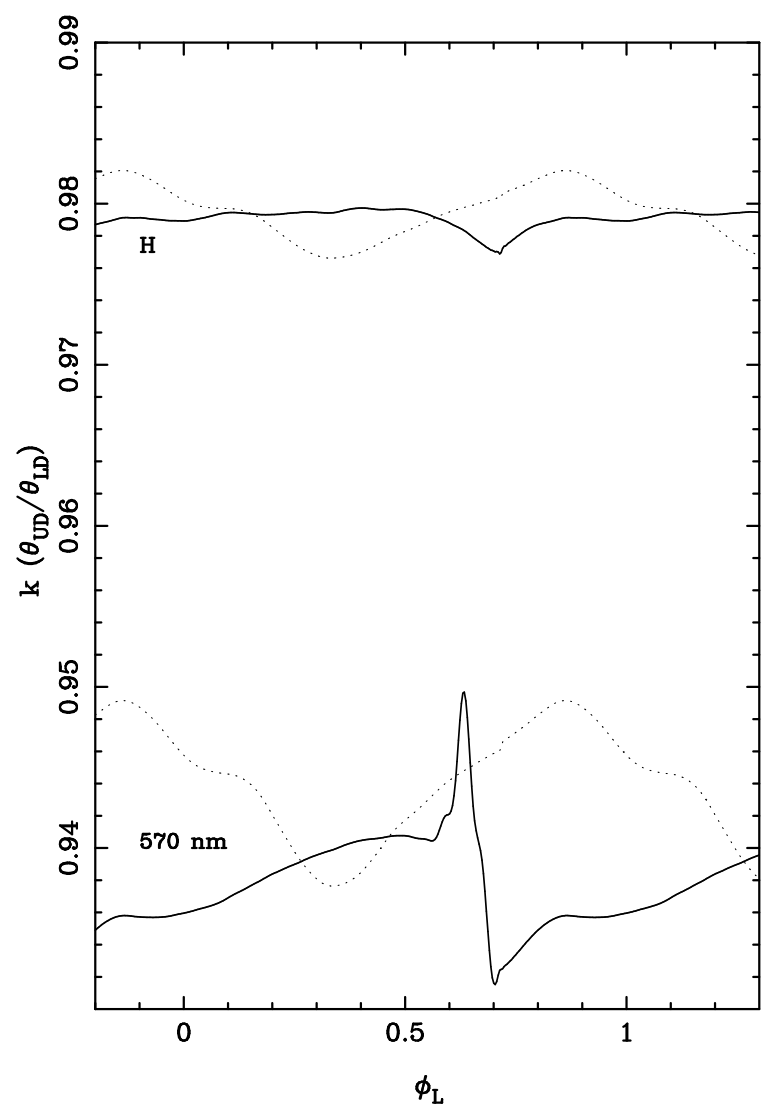

Figure 3. Phase dependent $k\left(\phi_{L}\right)$ for $\zeta$ Gem in the near-IR H-band and in the visible. The solid line is $k\left(\phi_{L}\right)$ from our hydrodynamic model, while the dotted line is derived from a hydrostatic model having $T_{\text {eff }}$ and $\log g$ from observations.

The unprecedented sensitivity of AMBER will result in high accuracy visibilities $\left(\sim 10^{-3}\right)$. This will allow the direct detection of the phase dependence of LD for nearby Cepheids. Figure 3 shows that the variation of the $\mathrm{LD}$ in the near-IR continuum is of the order of $0.2 \%$ for $\zeta$ Gem. AMBER will be able to detect these variations for similar Cepheids at a distance of 300-500 pc. The spectral capabilities of AMBER, coupled with the sensitivity offered by the Unit Telescopes (UT, providing an expected limiting magnitude $\mathrm{K} \sim 14$ for $R \sim 1,000$ ) will also allow to observe the LD in spectral lines, where the hydrodynamic effects are much larger.

The VLTI with AMBER will be the ideal instrument to directly measure the LD of pulsating Cepheids, and set strong constrains on the models. This will result in more accurate predictions of the LD corrections to use for the geometric BW method. The large aperture 
of the VLTI telescopes and the large maximum baseline will allow to detect the pulsation of a large sample of classical Cepheids. The limiting factor of the interferometers currently operating in the northern hemisphere is their limiting magnitudes (e.g. V $\sim 5$ for NPOI in the present configuration, and $\mathrm{K} \sim 4.5-5$ for the PTI). The $1.8 \mathrm{~m}$ aperture of the Auxiliary Telescopes (AT) and the $8 \mathrm{~m}$ diameter of the UT (necessary to resolve the spectral lines of Cepheids at $R \sim 1,000$ ) will extend the sample of Cepheids that can be observed in the southern hemisphere. Although the LD will not be directly measured for the most distant sources with the maximum $200 \mathrm{~m}$ baseline, the accurate predictions from the models calibrated with nearby Cepheids will still allow reliable distance determinations even when the stellar disk is not fully resolved. This in turn will result in a better calibration of the PL relation, and thus in a much improved distance scale from classical Cepheids.

\section{Acknowledgements}

This work was partially supported by NSF grant AST 98-76734. M.K. is a member of the Chandra Science Center, which is operated under contract NAS8-39073, and is partially supported by NASA.

\section{References}

Baade, W. 1926, Astron. Nachr., 228, 359

Feast, M.W., Catchpole, R.M. 1997, MNRAS, 286, L1

Kervella,P. Coudé du Foresto, V., Perrin, G., Schöller, M., Traub, W. A., Lacasse, M. G. 2001, A\&A, 367, 876

Krockenberger, M., Sasselov, D.D., Noyes, R.W. 1997, ApJ, 479, 875

Kurucz, R.L. 1970, SAO Special Rep. 309

Kurucz, R.L. 1979, ApJS, 40,1

Kurucz, R.L. 1993, in "Light-Curve Modeling of Eclipsing Binary Stars", ed. E.F. Milone (New York: Springer), 93

Lane, B., Kuchner, M.J., Boden, A.F., Creech-Eakman, M., Kulkarni, S.R. 2000, Nature, 407, 485

Lane, B.F., Creech-Eakman, M., Nordgren, T. 2002, ApJ, 573, 330

Leavitt, H.S. 1906, Ann. Astron. Harvard Coll. Obs., 60, 87

Madore, B.F., Freedman, W.L. 1998, ApJ, 492, 110

Marengo, M., Sasselov, D.D., Karovska, M.K., Papaliolios, C., Armstrong, J.T. 2002, ApJ, 567, 1131

Sasselov, D.D., Karovska, M. 1994, ApJ, 567, 1131

Sasselov, D.D., Lester, J.B. 1994, ApJ 423, 795

Wesselink, A. 1946, Bull. Astron. Inst. Netherlands, 10, 91 